\title{
Matériaux en couches minces: des corps purs composés aux méta-matériaux
}

\author{
Michel LEQUIME \\ Institut Fresnel, Equipe Couches Minces Optiques \\ michel.lequime@fresnel.fr
}

Le domaine des couches minces optiques a vécu ces 20 dernières années de profondes mutations [1], aussi bien au niveau des systèmes de dépôt lle développement des procédés dits énergétiques, tels que la pulvérisation ionique, donne aujourd'hui accès à des couches dépourvues de toute porosité et présentant nombre des caractéristiques d'un matériau massif) que dans celui du pilotage de ces procédés, spontanément très stables (le contrôle optique in situ permet en effet de réaliser des arrêts de couche avec une précision réellement sub-nanométrique). Mais, bien évidemment, de telles mutations ont aussi eu des impacts sur la nature et les caractéristiques des matériaux entrant dans la constitution de ces empilements complexes. C'est à cet aspect spécifiquement matériau que sera consacré le présent panorama.

\section{Les corps purs composés propriétés et mise en œuvre}

Deux classes de matériaux sont aujourd'hui utilisées de manière totalement maitrisée lors de la fabrication d'empilements de couches minces optiques : il s'agit en premier lieu des oxydes, ces diélectriques qui interviennent dans la constitution de la plupart des filtres optiques interférentiels, mais aussi de certains métaux, comme l'argent ou l'aluminium, dont l'utilisation s'avère souvent requise lorsque l'on s'intéresse à la réalisation de fonctions réfléchissantes (et/ou non réciproques, c'est-à-dire dépendant du sens de propagation de la lumière), et que les performances de celles-ci sont spécifiées sur de larges domaines de longueurs d'onde (la totalité du spectre visible, par exemple).

Parmi les oxydes les plus couramment utilisés, on retrouve bien évidemment la silice (ou dioxyde de silicium, de formule $\mathrm{SiO}_{2}$ ), qui reste un choix quasi obligé en terme de matériau bas indice $(\mathrm{n} \sim 1,47$ à $600 \mathrm{~nm}$ ), mais aussi le pentoxyde de niobium $\left(\mathrm{Nb}_{2} \mathrm{O}_{5}\right)$, qui a récemment supplanté le pentoxyde de tantale $\left(\mathrm{Ta}_{2} \mathrm{O}_{5}\right)$ comme matériau haut indice, car il présente un indice plus élevé $\left(2,35\right.$ pour $\mathrm{Nb}_{2} \mathrm{O}_{5}$ à comparer à 2,15 pour $\mathrm{Ta}_{2} \mathrm{O}_{5}$ ) sans que cela ne s'accompagne des difficultés de mise en œuvre qui sont celles du dioxyde de titane $\mathrm{TiO}_{2}$ et qui sont liées à la présence simultanée de deux phases structurales (rutile et anatase) de propriétés optiques sensiblement différentes ( $n \sim 2,2$ et 2,7 ). On citera également le dioxyde d'hafnium $\mathrm{HfO}_{2}$ ( $n$ 2,05), dont l'usage s'impose, en association avec la silice, lorsque l'on a pour objectif la réalisation d'une fonction de filtrage dans le domaine compris entre le bleu profond et le proche ultra-violet (typiquement entre 300 et $450 \mathrm{~nm}$ ).

Pour appréhender de manière correcte les caractéristiques d'une fonction de filtrage optique, et donc les spécifications de performances qui vont s'appliquer aux matériaux entrant dans sa réalisation, il semble ici nécessaire de rappeler quelques notions importantes:

- L'indice de réfraction d'un milieu est en fait une quantité complexe $(n+i \kappa)$, I'usage étant de désigner sous l'appellation (un peu abusive) d'indice la seule partie réelle $\mathrm{n}$ et d'appeler coefficient d'extinction sa partie imaginaire $\kappa$. L'indice $n$ va avoir essentiellement un impact sur le nombre de couches nécessaires à la réalisation d'une fonction de filtrage donnée, au travers du rapport entre l'indice $\mathrm{nH}$ du matériau de haut indice et celui $\mathrm{nB}$ du matériau de bas indice qui interviennent conjointement dans la formule de cet empilement. Plus ce rapport $\mathrm{nH} / \mathrm{nB}$ sera grand (on parlera ici de choc d'indice), et plus faible sera le nombre de couches entrant dans la formule de cet empilement à propriétés optiques équivalentes. C'est essentiellement pour cette raison que l'on cherchera à disposer de matériaux en couches minces présentant des indices de réfraction très contrastés et c'est ce qui justifie, dès que les contraintes de compatibilité chimique ou de transparence le permettent, l'emploi de matériaux tels que le bifluorure de magnésium $\left(\mathrm{MgF}_{2}, \mathrm{n} \sim 1,38\right.$ à $\left.600 \mathrm{~nm}\right)$ ou le silicium (Si, $\mathrm{n} \sim 3,45$ à $1500 \mathrm{~nm}$ ).

- La partie imaginaire $k$ de cet indice de réfraction va quant à elle définir l'absorption des différentes couches constitutives de l'empilement et donc son niveau global de pertes. C'est là qu'intervient l'une des particularités les plus importantes des filtres optiques interférentiels, à savoir le phénomène d'exaltation du champ électrique dans l'épaisseur de certaines couches, dites résonantes. Ce phénomène trouve son origine dans la sommation, constructive ou destructive, des nombreuses ondes, progressives et rétrogrades, qui prennent naissance sur les différentes interfaces de l'empilement et qui peuvent conduire à 
ce que l'intensité $E^{2}$ de ce champ soit localement multipliée par un facteur compris entre quelques unités et plusieurs milliers. Or l'absorption du composant est pilotée par le produit $\mathrm{K}^{2}$ entre cette intensité locale et le coefficient d'extinction de la couche. II sera donc indispensable, malgré les faibles épaisseurs caractéristiques des couches utilisées, de disposer de matériaux présentant de très faibles $a b$ sorptions, et donc de très faibles parties imaginaires к. À titre d'exemple, les coefficients d'extinction des oxydes évoqués plus haut ne dépassent pas quelques $10^{-6}$, ce qui revient à dire qu'une simple perte de $10 \%$ serait associée à l'emploi d'une couche non résonante de plus de 5 millimètres d'épaisseur, soit l'équivalent de quelques 70000 couches quart d'onde ! - Cette contrainte de faible absorption est aujourd'hui devenue réellement critique, car le nombre de couches intervenant dans la réalisation des fonctions complexes de filtrage que spécifient des secteurs d'application tels que les télécommunications optiques, le biomédical, l'observation astronomique ou le spatial, a littéralement explosé, passant en moins de quinze ans de quelques dizaines à plusieurs centaines [1], sans que les niveaux admissibles de pertes soient en rien relâchés, bien au contraire : les transmissions maximales spécifiées dans la partie passante du spectre excédent en effet couramment $90 \%$, comme on peut le constater, à titre d'exemple, sur le graphe présenté à la figure 1 et qui reproduit les résultats d'une mesure expérimentale de transmission réalisée sur un filtre complexe comprenant environ une centaine de couches et dont la bande passante est centrée vers $750 \mathrm{~nm}$ (la face arrière du substrat est ici non traitée).

- On rappelle enfin que la conservation de l'énergie à la traversée d'un composant optique (et les filtres optiques interférentiels n'échappent bien évidemment pas à cette règle) s'écrit, en toute généralité :

$$
R+T+A+D=1
$$

où $R$ et $T$ désignent les coefficients de réflexion et de transmission en énergie duabsorption et $\mathrm{D}$ celui associé à la diffusion de la lumière. On comprendra, à la lecture des paragraphes qui précèdent, qu'une fois résolu le problème de l'absorption, ce sont

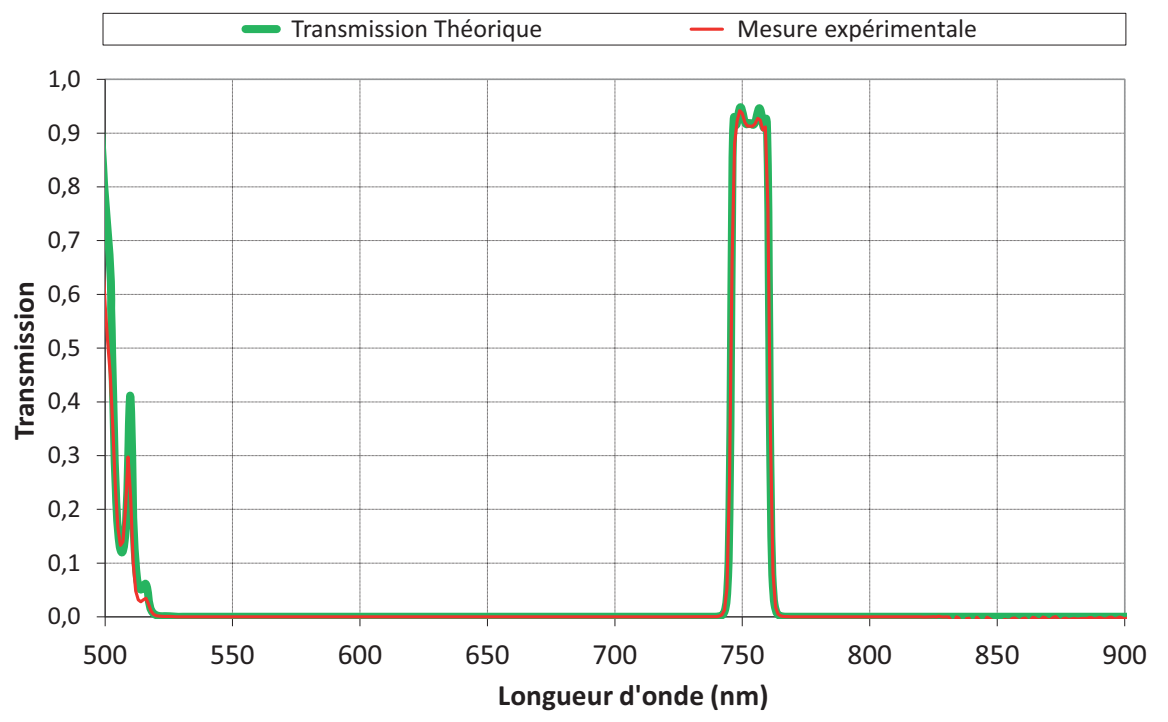

Figure 1. Transmission spectrale d'un filtre interférentiel comportant une centaine de couches minces (en trait épais vert, calcul théorique ; en trait fin rouge, mesure expérimentale).

les mécanismes de diffusion prenant naissance dans l'empilement de couches qui peuvent constituer le dernier facteur limitant, en dégradant le contraste des images délivrées par l'instrument dans lesquels ces empilements interviennent.

- Or, deux phénomènes sont responsables de cette diffusion : il s'agit de la rugosité des interfaces entre couches d'une part, et de l'hétérogénéité volumique de ces différentes couches d'autre part. Pour minimiser le premier, il convient d'utiliser des substrats super-polis et d'avoir recours à des procédés de dépôt dits énergétiques, dont on sait qu'ils conduisent à la croissance de couches dont la rugosité de l'interface supérieure reproduit presque intégralement celle de l'interface inférieure. Pour réduire les effets du second, il faut sélectionner des procédés permettant d'approcher en couches minces les performances ultimes du matériau massif : là encore, le recours à des procédés énergétiques, comme la pulvérisation ionique, semble aujourd'hui s'imposer. II n'en reste pas moins vrai que les effets d'exaltation du champ électrique évoqués plus haut dans le cas de l'absorption s'appliquent de la même manière aux mécanismes de diffusion par des interfaces rugueuses ou des volumes hétérogènes, et qu'ils peuvent donc conduire à des résonances en diffusion dont la densité spectrale et angulaire de luminance ne saurait être ignorée au moment de la conception d'instruments modernes.

\section{Des corps purs composés aux mélanges}

Le paragraphe précédent consacré aux corps purs composés a permis de souligner la grande maitrise qui préside aujourd'hui à leur dépôt, mais il a aussi mis en lumière la palette relativement restreinte dont dispose le concepteur de filtres interférentiels en termes de valeurs d'indice.

C'est là que les procédés de co-pulvérisation interviennent en rendant accessible n'importe quelle valeur intermédiaire d'indice située entre les deux valeurs extrêmes qui correspondent à des matériaux purs.

Pour illustrer cette capacité nouvelle à déposer des mélanges, nous avons choisi le cas particulier de la machine de dépôt HELIOS [2], développée par Leybold Optics au milieu des années 2000 et dont I'Institut Fresnel s'est doté en novembre 2012. II s'agit d'une machine de pulvérisation magnétron (les ions présents dans la chambre de dépôt sont accélérés et leurs trajectoires courbées vers une cible métallique par un champ magnétique) avec assistance plasma réactive (l'oxydation des couches métalliques sous-stœchiométriques est réalisée par un plasma d'ions oxygène), procédé qui est habituellement désigné sous l'acronyme générique de PARMS (plasma assisted reactive magnetron sputtering).

La figure 2 représente de manière schématique la structure de cette chambre de dépôt. Les pièces à traiter sont disposées 


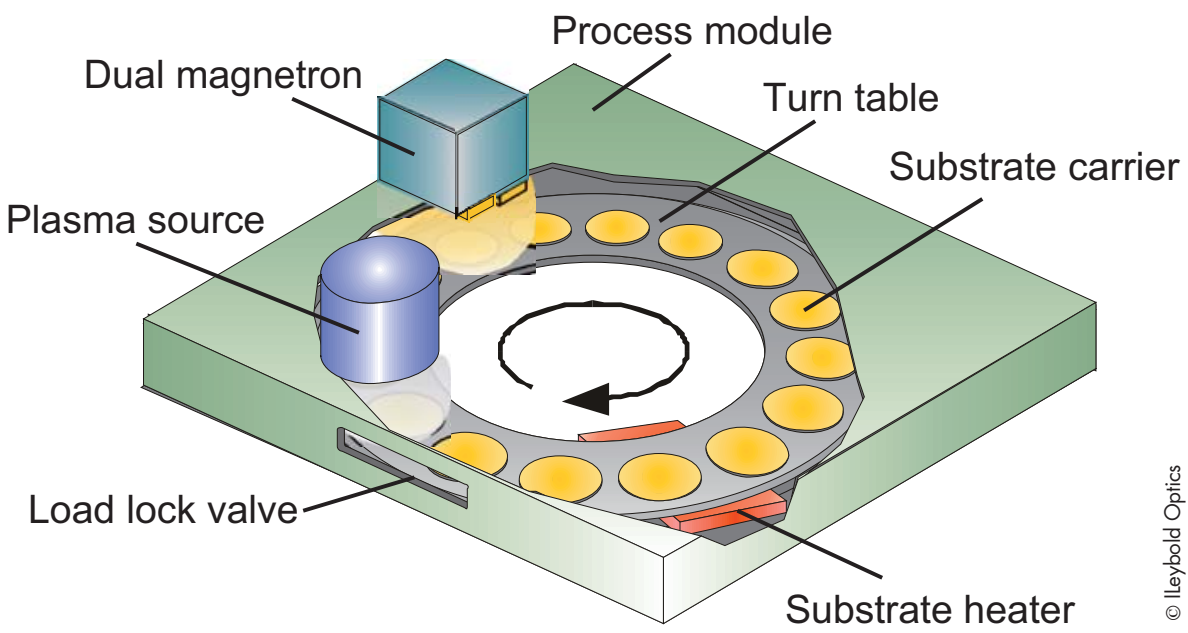

Figure 2. Schéma de principe du fonctionnement de la machine de dépôt HELIOS, utilisant la pulvérisation magnétron avec assistance plasma réactive.

dans des logements régulièrement répartis sur un plateau tournant à grande vitesse (240 tours par minute). À l'occasion de chaque tour de plateau, ces pièces passent successivement devant un premier magnétron équipé par exemple d'une cible de niobium, puis devant un second doté par exemple d'une cible de silicium. Durant leur bref passage devant l'un ou l'autre de ces magnétrons, la surface de ces pièces se couvre d'une fine couche de matériau métallique partiellement oxydé (la chambre est en effet sous atmosphère réactive d'oxygène), dont l'épaisseur instantanée est de l'ordre de grandeur de l'angström. Comme

indiqué plus haut, cette fine couche est ensuite totalement oxydée par la source plasma d'assistance. Il suffit donc de régler la puissance de commande appliquée à chacun des deux magnétrons pour passer continument d'une couche pure de $\mathrm{SiO}_{2}$ à une couche pure de $\mathrm{Nb}_{2} \mathrm{O}_{5}$ en transitant par toutes les compositions possibles de mélanges, de formule $\left[\mathrm{SiO}_{2}\right]_{x}\left[\mathrm{Nb}_{2} \mathrm{O}_{5}\right]_{1-x}$ et donc tous les indices de réfraction associés, comme représenté à la figure 3 .

Ce procédé permet donc de disposer d'une large gamme d'indices synthétiques et de rendre ainsi possible le développement d'une nouvelle classe

Refractive index vs. power ratio of reactive co-sputtered $\mathrm{Nb}_{\mathrm{x}} \mathrm{Si}_{\mathrm{y}} \mathrm{O}_{z}$ layers

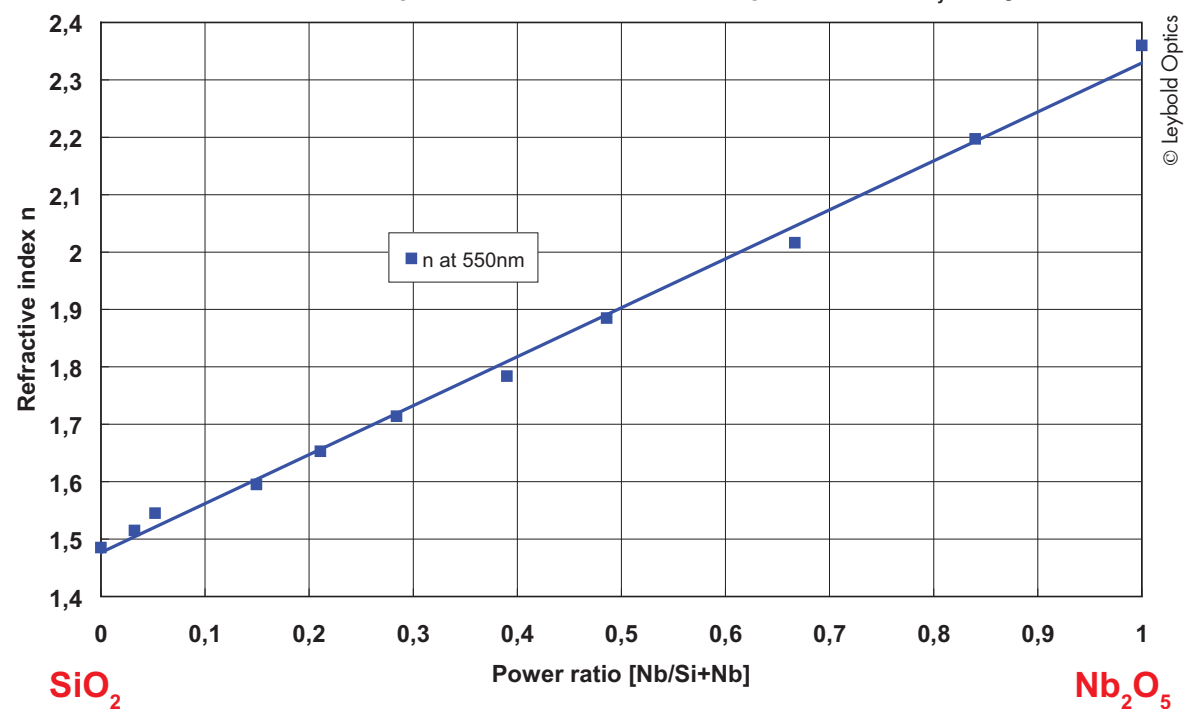

Figure 3. Evolution de l'indice de réfraction d'un mélange $\mathrm{Nb}_{2} \mathrm{O}_{5} / \mathrm{SiO}_{2}$ en fonction du rapport des puissances de commande appliquées aux deux magnétrons. acal $\left.\right|^{\text {bfi }}$

European leader in advanced technology solutions

\section{SOLUTIONS REMOTE SENSING}

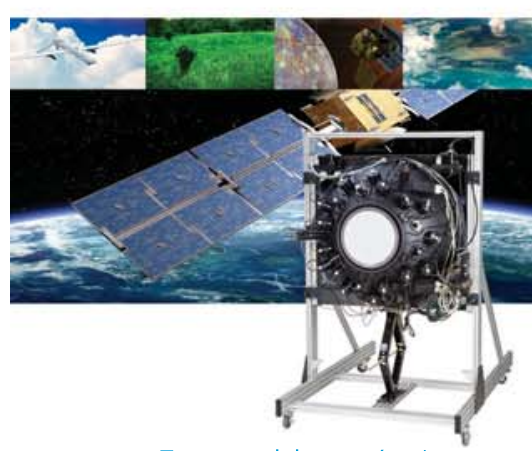

Tests au sol des caméras/senseurs et imageurs hyperspectraux - Tests de capteurs CCD/CMOS avec ou sans optiques
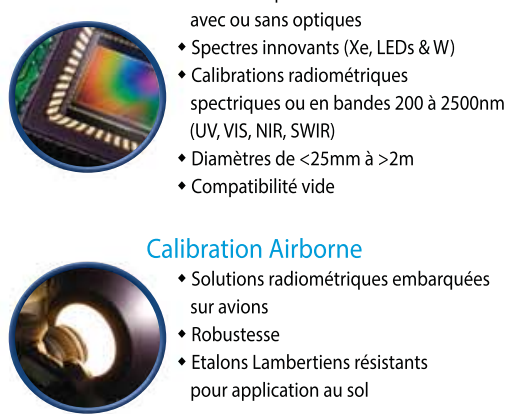

Spectralon ${ }^{\circledast}$ qualifié spatial - Processus de fabrication spécifique pour éliminer la dégradation aux UV - Stabilité dans le temps

- Fort coefficient de réflexion de 250 nm à 2500 nm

- Utilisation au sol et en orbite

Solutions de mesures d'efficacité

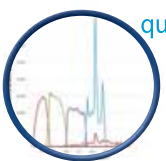

uantique

- Sources uniformes en éclairement

- Tests d'efficacité quantique spectrale

- Champ plan, linéarité, dynamique

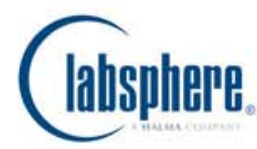

Présents sur OPTRO 2014 stand 24

Nous vous invitons à découvrir

nos solutions Remote Sensing

Pour plus d'informations

N'hésitez pas à solliciter nos ingénieurs Tel +33 (0)1 60795938 photonique.fr@acalbfi.fr

www.acalbfi.fr 
d'empilements dits à pseudo-gradient, pour lesquels les valeurs d'indice utilisées conservent, quoique nombreuses, un caractère résolument discret (le jeu d'indices employé peut comprendre entre 3 et 11 valeurs) ; cette appellation de pseudo-gradient a été introduite en référence aux empilements de type gradient (ou rugate) où la variation du profil d'indice est, cette fois, de nature continue.

II n'en reste pas moins vrai que le contraste d'indice de ces empilements pseudo-gradients est entièrement régi par celui des matériaux purs. Pour augmenter ce contraste, il faut donc envisager de réaliser un mélange faisant intervenir un nouveau corps pur, si possible d'indice beaucoup plus faible et c'est ce que réalisent dans une certaine mesure les matériaux poreux, où ce nouveau corps pur n'est rien d'autre que de l'air.

\section{Les matériaux poreux : un cas particulier de mélange}

La motivation à disposer d'un matériau synthétique d'indice faible trouve son origine dans le cas des traitements antireflets qui sont spécifiés sur un large domaine de longueurs d'onde. On montre en effet que les performances d'un tel empilement sont, à nombre égal de couches, d'autant meilleures que le choc d'indice est fort et que l'indice de la dernière couche est faible.

C'est, comme évoqué plus haut, la raison de l'emploi fréquent du bifluorure de magnésium comme couche terminale d'un empilement antireflet, même si toutes les autres couches de cet empilement font appel à des oxydes.

De nombreuses méthodes ont été envisagées pour obtenir des matériaux poreux de grande qualité, et il n'est évidemment ni possible, ni réellement utile, d'en faire ici une présentation exhaustive. Nous nous limiterons donc à faire état de la démarche adoptée par Nikon Corporation [3], qui a conduit cette société à utiliser le dépôt, par procédé sol-gel, d'une couche composée de nano-particules de $\mathrm{MgF}_{2}$ de diamètre compris entre 5 et $10 \mathrm{~nm}$. L'indice équivalent de la couche ainsi réalisée est de l'ordre de 1,26 (à comparer à 1,38 dans le cas d'une couche non poreuse) et l'emploi de ce matériau très bas indice comme couche terminale (épaisseur 104,6 nm) d'un empilement antireflet composé de 8 couches alternées $\mathrm{SiO}_{2} / \mathrm{Nb}_{2} \mathrm{O}_{5}$ permet $d$ 'abaisser le coefficient de réflexion d'un verre $d^{\prime}$ indice 1,59 à moins de $0,03 \%$ sur l'ensemble du domaine spectral compris entre 430 et $675 \mathrm{~nm}$.

\section{Vers l'emploi de méta-matériaux?}

Le simple exemple des couches antireflets montre l'importance qu'il peut y avoir à augmenter la gamme d'indices de réfraction accessibles, et l'on comprend aisément l'intérêt qu'a suscité de ce point de vue l'émergence toute récente des méta-matériaux, ces matériaux artificiels qu'une structuration à l'échelle nanométrique a doté de fascinantes propriétés d'indice (comme des indices nuls ou des indices négatifs).

Et même s'il est clair qu'il reste encore un long chemin pour que ces structures soient intégrées à la boîte à outils du concepteur de filtres optiques interférentiels [4], il aurait été regrettable de ne pas donner dans ce panorama des matériaux en couches minces un exemple de réalisation faisant appel à ce type d'approche.

L'exemple que nous avons retenu est un filtre passe-bande de $500 \mathrm{~nm}$ de large, centréà $3,25 \mu \mathrm{m}$ [5]. Il a été récemment développé par l'Université de Pennsylvanie (Departement of Electrical Engineering and (enter for Nanoscale Science), et ne comprend en fait que 3 couches, à savoir une couche centrale de polyimide de 450 nm d'épaisseur entourée de deux fines couches d'or de $30 \mathrm{~nm}$ chacune. Cet empilement minimal est gravé dans
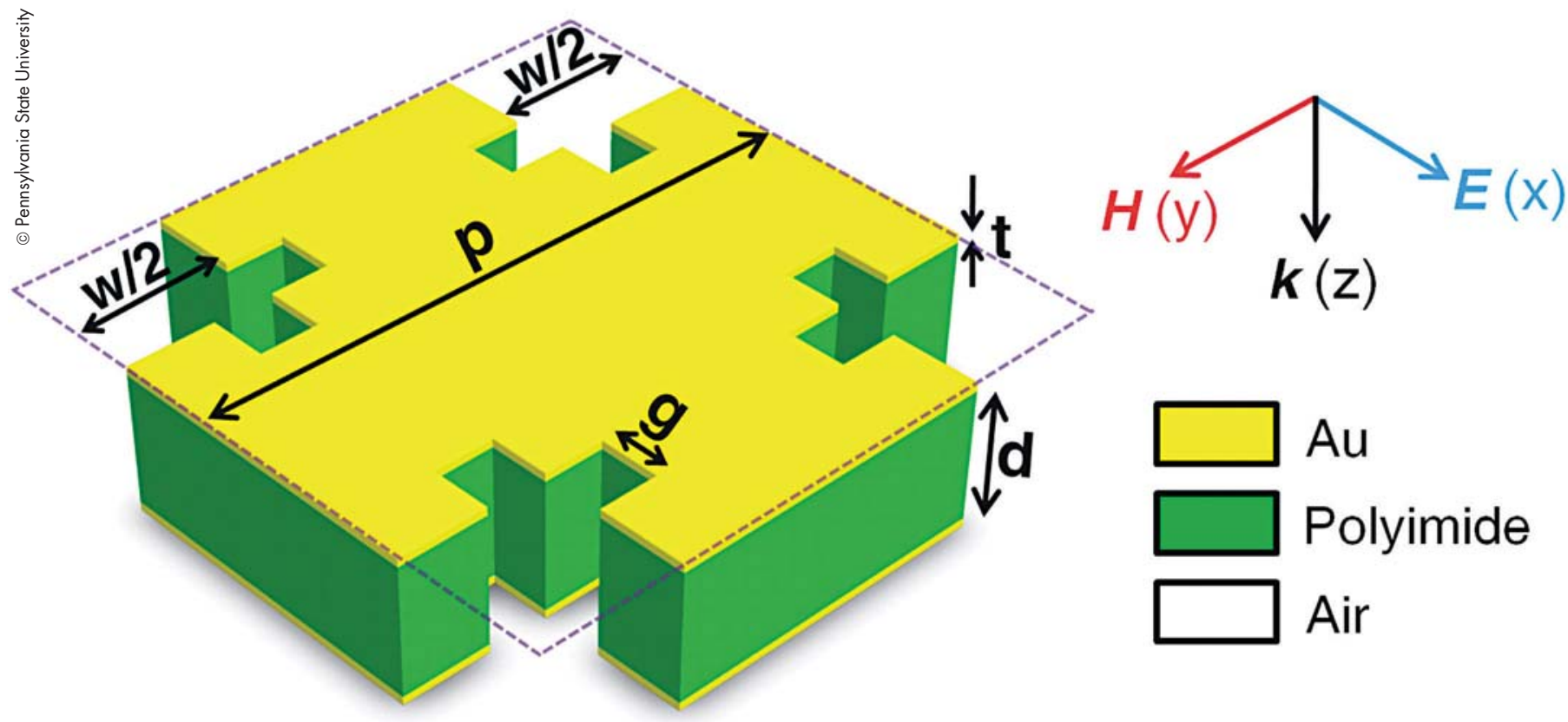

Figure 4. a. Représentation schématique de la cellule élémentaire de la structure en résille gravée dans un empilement or/polyimide/or $(p=2113 \mathrm{~nm}, \mathrm{w}=990 \mathrm{~nm}$, $g=198 \mathrm{~nm}, \mathrm{t}=30 \mathrm{~nm}$ et $d=450 \mathrm{~nm})$. 


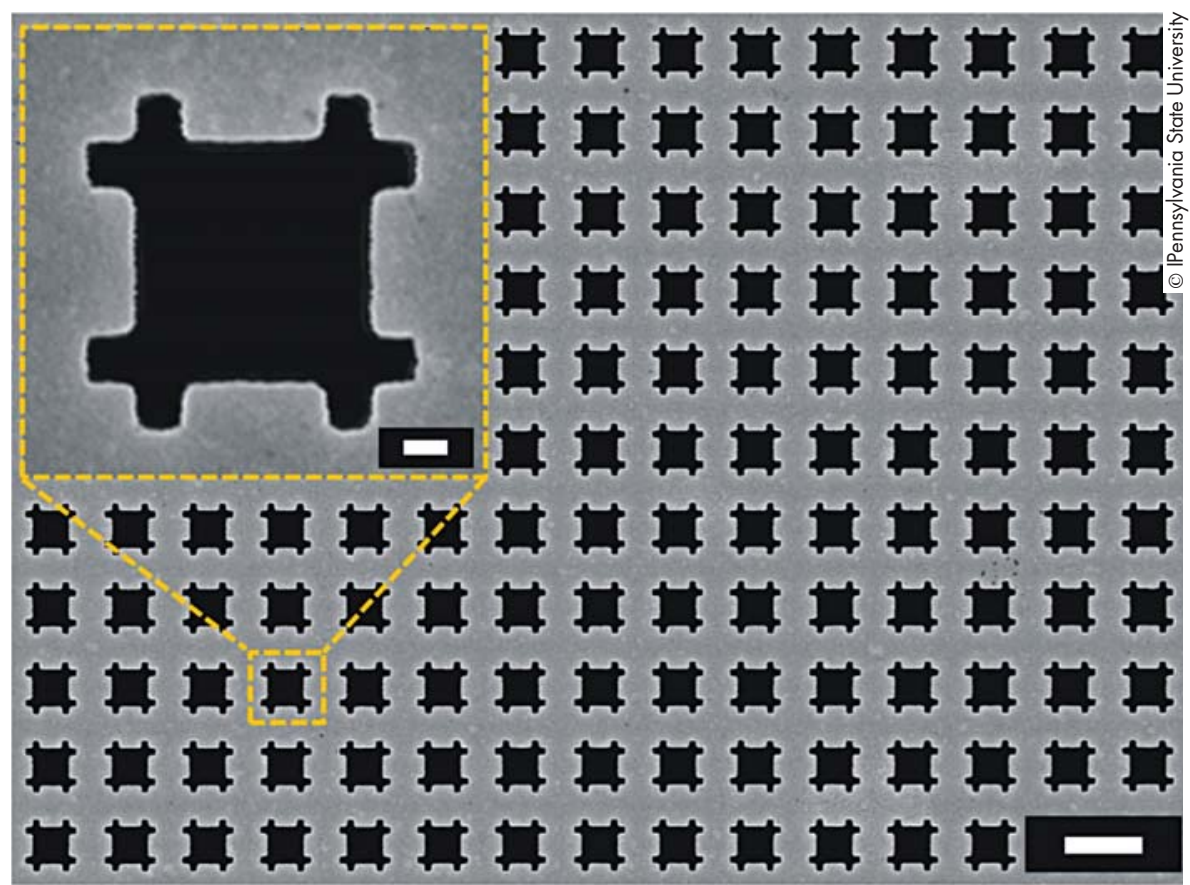

Figure 4. b. Image par microscopie électronique à balayage de la structure de filtrage autoporteuse obtenue par réplication du motif élémentaire décrit à la Figure $4 a$ (barre d'échelle : $3 \mu \mathrm{m})$. L'encart présente une vue agrandie du motif élémentaire (barre d'échelle $200 \mathrm{~nm}$ ).

toute son épaisseur par un motif en résille dont la géométrie, finement optimisée, est représentée à la figure $4 \mathrm{a}$. La figure $4 \mathrm{~b}$ permet de mieux se rendre compte de la structuration de ce filtre que l'on qualifiera d'auto-porteur, puisqu'il n'est pas déposé comme à l'accoutumée sur un substrat massif, mais simplement tendu sur un cadre en aluminium.

La transmission dans la bande de ce filtre est légèrement supérieure à $80 \%$, tandis que le niveau de réjection hors bande (entre 2,5 et $4 \mu \mathrm{m}$ ) est de l'ordre de $-10 \mathrm{~dB}$. Cette dernière caractéristique peut sembler quelque peu en retrait des autres performances, mais il ne faut pas perdre de vue que cet empilement a une épaisseur hors tout de $510 \mathrm{~nm}$, ce qui est une valeur absolument remarquable si on la compare à celle de la longueur d'onde centrale du filtre ainsi réalisé.

\section{Conclusion}

Le lecteur aura, nous l'espérons, perçu, à la lecture de cette revue très synthétique, et forcément incomplète, des matériaux en couches minces, le dynamisme toujours renouvelé de cette thématique industrielle diffusante et son enracinement spontané dans le monde des nanotechnologies, qu'elle a contribué à créer et qu'elle accompagne dans son déploiement.

\section{Références}

[1] M. Lequime et M.-F. Ravet-Krill, Les couches minces optiques : entre recherche appliquée et révolution industrielle, Photoniques 42, 51-53 (2009)

[2] H. Hagedorn, W. Lehnert, J. Pistner, M. Scherer, A. Zöller, Plasma Assisted Reactive Magnetron Sputtering of Demanding Interference Filters, Proceedings of the 55th Annual Technical Conference of the Society of Vacuum Coaters

[3] T. Murata, H. Ishizawa, A. Tanaka, Highperformance antireflective coatings with a porous nanoparticle layer for visible wavelengths, Appl. Opt. 50, C403-C407 (2011)

[4] M. Lequime, B. Gralak, S. Guenneau, M. Zerrad, C. Amra, Negative indices and the admittance formalism in multilayer optics, in Optical Interference Coatings Conference (Optical Society of America, 2013), paper TB9

[5] Z.H. Jiang, S. Yun, L. Lin, J.A. Bossard, D.H. Werner, T.S. Mayer, Tailoring dispersion for broadband low-loss optical metamaterials using deep-subwavelength inclusions, Scientific Reports 3, 01571 (2013) (2012), 332-335

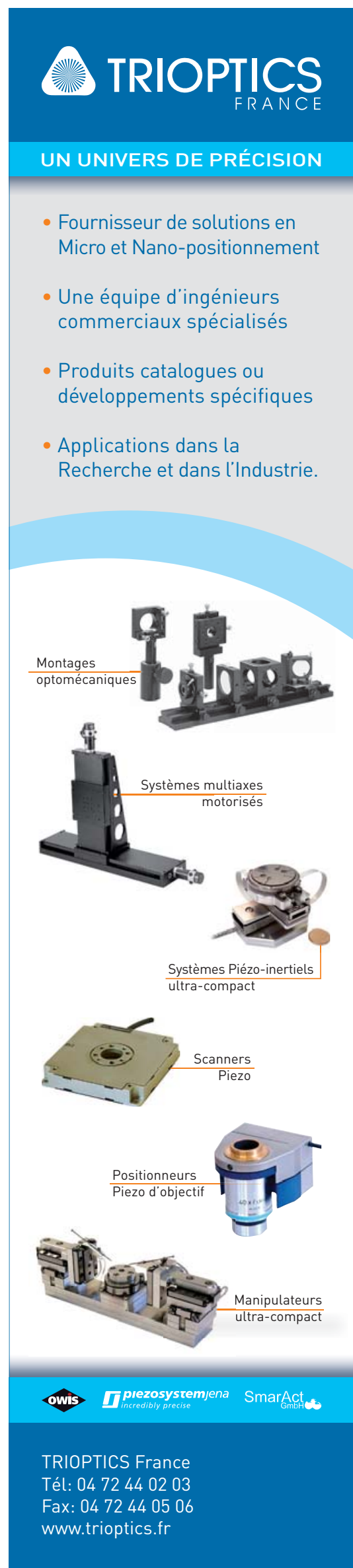

0
0
$\frac{0}{0}$
$\frac{0}{5}$
$\frac{0}{0}$
$\frac{0}{2}$ 